\title{
On the very weak solvability of the beam equation
}

\author{
J.I.Díaz
}

November 26, 2010

\begin{abstract}
We get some necessary and sufficient conditions for the very weak solvability of the beam equation stated in terms of powers of the distance to the boundary, accordingly to the boundary condition under consideration. We get a $L^{1}$-estimate by using an abstract result due to Crandall and Tartar. Applications to some nonlinear perturbed equations and to the eventual positivity of the solution of the parabolic problems are also given.
\end{abstract}

\section{Introduction}

The main goal of this Note is to present some new results proving that in the case of higher order equations and Dirichlet boundary conditions the class of $L_{L o c}^{1}(\Omega)$ data for which the existence and uniqueness of a very weak solution can be obtained is larger than $L^{1}(\Omega, \delta)$, where $\delta=\operatorname{dist}(x, \partial \Omega)$ (the optimal class for the case of second order equations such as it was originally proved by Haim Brezis, in the seventies, in a famous unpublished manuscript (see also [4], [9], [10] and [11])). For instance, for some stationary onedimensional linear 4th-order equations we shall prove here that the optimal class of data is the space $L^{1}\left(\Omega, \delta^{2}\right)$. Moreover we shall analyze the optimal solvability also for the case of other boundary conditions: something which, as far as we know, was not considered before in the literature (even for the case of second order equations).

In some sense, the obtained results give an answer to the question about of the greatest weight profile which can support a simple beam such that its two extremes are horizontally supported (for instance to a wall) and do not experience any deflection.

We point out that although we shall deal with the Euler-Bernoulli beam model, but most of the results remain valid for equations of order $2 m, m \in \mathbb{N}$. In a first part we shall consider the stationary case:

$$
(S P) \begin{cases}\frac{d^{4} u}{d x^{4}}=f(x) & x \in \Omega=(0, L), \\ \text { +boundary conditions }(B C) . & \end{cases}
$$

With respect to the beam modelling, we are assuming here $I_{z} E=1$ but, again, it is only for simplicity. Here $I_{z}$ represents the moment of inertia with respect 
to $z$ axes and $E$ is the Young's modulus constant. $(B C)$ corresponds to two sets of two identities (two at $x=0$ and another two at $x=L$ ) among the following possibilities

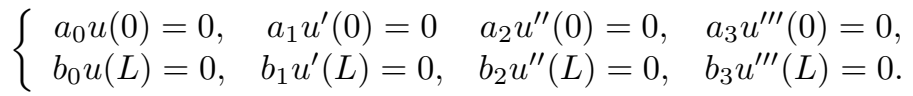

Here the coefficients are taken such that $a_{i}, b_{i} \in\{0,1\}$ and $\sum a_{i}=2, \sum b_{i}=2$. Dirichlet conditions (clamped beam) corresponds to

$$
(D B C) \begin{cases}u(0)=0, & u(L)=0 \\ u^{\prime}(0)=0 & u^{\prime}(L)=0\end{cases}
$$

If the beam is simply supported at its extremes (hinged beam) it corresponds to $a_{0}=b_{0}=a_{2}=b_{2}=1$ (and so $a_{1}=b_{1}=a_{3}=b_{3}=0$ ). Neumann type boundary conditions obey to different choices but assuming always that $a_{0}=$ $b_{0}=0$. Finally, a very often considered situation corresponds to a cantilever bar $\left(x=0\right.$ clamped and $x=L$ free): $a_{0}=a_{1}=b_{2}=b_{3}=1$. It turns out that in any of the above mentioned problems the optimal weight $w(x)$ for the solvability is $\delta_{\mathbf{a b}}(x)=\max \left\{\left(a_{0} \wedge a_{1}\right) d(x, 0)^{2},\left(a_{0} \wedge a_{2}\right) d(x, 0), a_{3}\right\} \max \left\{\left(b_{0} \wedge\right.\right.$ $\left.\left.b_{1}\right) d(x, L)^{2},\left(b_{0} \wedge b_{2}\right) d(x, L), b_{3}\right\}$, where $c \wedge d=\min (c, d)$. For instance, for the Dirichlet problem, $[\mathbf{a}=(1,1,0,0), \mathbf{b}=(1,1,0,0)]$, we must take $\delta_{\mathbf{a b}}(x) \sim \delta^{2}(x)$ with $\delta=\operatorname{dist}(x, \partial \Omega)$.

In a second part of the Note we shall consider a nonlinearly perturbed problem and the associated parabolic equation. Some other applications will be given in and [8].

\section{Necessary and Sufficient conditions for the ex- istence of solutions for the stationary prob- lem.}

To fix ideas I will consider now the case of Dirichlet boundary conditions.

Definition. Given $f \in L_{L o c}^{1}(0, L)$ a function $u \in L_{L o c}^{1}(0, L)$ is a solution of $(S P)$ in $D^{\prime}(0, L)$ if $\left\langle u, \frac{d^{4} \zeta}{d x^{4}}\right\rangle_{D^{\prime} D}=\langle f, \zeta\rangle_{D^{\prime} D}$ for any $\zeta \in D(0, L)=C_{c}^{\infty}(0, L)$.

We introduce now the space $V$ associated to the boundary $(B C)$ as the closure in $W^{4, \infty}(0, L)$ of the set $\left\{\zeta \in C^{4}([0, L]): \zeta\right.$ satisfies $\left.(B C)\right\}$. For instance, for the case of Dirichlet boundary conditions $V=W^{4, \infty}(0, L) \cap W_{0}^{2, \infty}(0, L)$.

Definition. Given $f \in L^{1}\left(0, L: \delta_{\mathbf{a b}}\right)$ a function $u \in L^{1}(0, L)$ is a "very weak solution" of $(S P)$ and $(B C)$ if for any $\zeta \in V$

$$
\int_{0}^{L} u(x) \frac{d^{4} \zeta}{d x^{4}}(x) d x=\int_{0}^{L} f(x) \zeta(x) d x .
$$

Notice that $\zeta \in V$ implies that $|\zeta(x)| \leq c \delta_{\mathbf{a b}}$ for any $x \in(0, L)$ and so the above identity is well justified. 
The main result of this Note is the following:

Theorem 1. a) Sufficiency. Assume that $a_{2} a_{3}=0$ if $b_{2}=b_{3}=1$ (respectively, $b_{2} b_{3}=0$ if $\left.a_{2}=a_{3}=1\right)$. Then, for any $f \in L^{1}\left(0, L: \delta_{\mathbf{a b}}\right)$ there exists a unique very weak solution of $(S P)$ and $(B C)$. Moreover we have the estimate (weak maximum principle)

$$
C\left\|u_{+}\right\|_{L^{1}(0, L)} \leq\left\|f_{+}\right\|_{L^{1}\left(0, L: \delta_{\mathbf{a b}}\right)}
$$

for some $C>0\left(C=24 L^{4}\right.$ for $\left.(D B C)\right)$ where, in general, $h_{+}=\max (0, h)$. Moreover $u \in C^{3}([0, L])$.

b) Strong maximum principle. Let $f \in L^{1}\left(0, L: \delta_{\mathbf{a b}}\right)$ with $f \geq 0$ a.e. $x \in(0, L)$, $f \neq 0$. Then the very weak solution satisfies $u(x) \geq C\|f\|_{L^{1}\left(0, L: \delta_{\mathbf{a b}}\right)} \delta_{\mathbf{a b}}(x)>0$ for any $x \in(0, L)$, for some $C>0$ independent of $f$.

c) Necessity. Assume that $f \in L_{\text {Loc }}^{1}(0, L)$, such that $f \geq 0$ a.e. $x \in(0, L)$. Then if $\int_{0}^{L} f(x) \delta_{\mathbf{a b}}(x) d x=+\infty$ it cannot exist any $u \in C^{3}([0, L])$ satisfying $(B C)$ being also solution in $D^{\prime}(0, L)$ of $(S P)$.

Idea of the proof of the Theorem 1. For the existence part it is enough to use the Green function associated to the boundary conditions (see,e.g., [19]). Indeed, the expression $u(x)=\int_{0}^{L} G(x, y) f(y) d y$ is well justified since we have that $|G(., y)| \leq C \delta_{\mathbf{a b}}(y)$. For the proof of the $L^{1}$-estimate we shall use some "conservation formula". For the case of $(D B C)$ (for other boundary conditions the arguments are similar) we have:

Lemma 1. Let $f \in L^{1}\left(0, L: \delta^{2}\right)$ and let $u$ be any very weak solution of $(S P)$ and $(D B C)$. Then $24 L^{4} \int_{0}^{L} u(x) d x=\int_{0}^{L} x^{2}(L-x)^{2} f(x) d x$.

We also know (see $([6]))$ that if $f \in L_{\text {loc }}^{1}(0, L), f \geq 0$ on $(0, L)$ then $u(x) \geq 0$ for any $x \in(0, L)$. The last ingredient, to prove the $L^{1}$-estimate is an abstract result applied usually to hyperbolic equations.

Lemma 2 ([7]). Let $X, Y$ two vector lattices and $\lambda_{X}, \lambda_{Y}$ be nonnegative linear functionals on $X$ and $Y$ respectively. Let $C \subseteq X$ and $f, g \in C$ imply $f \vee g \in C$. Let $T: C \rightarrow Y$ satisfy $\lambda_{X}(f)=\lambda_{Y}(T(f))$ for $f \in C$. Then $(a) \Rightarrow(b) \Rightarrow(c)$ where $(a),(b),(c)$ are the properties:(a) $f, g \in C$ and $f \leq g$ imply $T(f) \leq T(g),(b) \lambda_{Y}\left((T(f)-T(g))_{+}\right) \leq \lambda_{X}\left((f-g)_{+}\right)$for $f, g \in C$, (c) $\lambda_{Y}(|T(f)-T(g)|) \leq \lambda_{X}(|f-g|)$.Moreover, if $\lambda_{Y}(F)>0$ for any $F>0$, then $(a),(b),(c)$ are equivalent.

Now, to prove the $L^{1}$-estimate $(1)$ we take $C=X=L^{1}\left(0, L: \widehat{\delta}^{2}\right), Y=$ $L^{1}(0, L), \lambda_{X}(f)=\int_{0}^{L} x^{2}(L-x)^{2} f(x) d x, \lambda_{Y}(F)=\int_{0}^{L} F(x) d x$ and $T(f)=24 L^{4} u$ (with $u$ the very weak solution of $(S P)$ and $(D B C)$ ). Then the identity of Lemma 2 coincides with the one in Lemma 1. So we get (b) of Lemma 2 which is the wanted $L^{1}$-estimate.

The proof of the strong maximum principle uses the estimate $|G(., y)| \leq C \delta_{\mathbf{a b}}(y)$. To prove part c), and more specifically the complete blow up (in the whole interval $(0, L))$ when $f \notin L^{1}\left(0, L: \delta_{\mathbf{a b}}\right)$ we truncate $f$ generating $f_{n}(x)=$ $\min (f(x), n)$. Now, if $u_{n}$ is the associated solution $\left(f_{n} \in L^{\infty}(0, L) \subset L^{1}(0, L\right.$ : $\left.\delta_{\mathbf{a b}}\right)$ ) then $u_{n}(x) \geq C\left\|f_{n}\right\|_{L^{1}\left(0, L: \delta_{\mathbf{a b}}\right)} \delta_{\mathbf{a b}}(x)$, which implies that $u_{n}(x) \nearrow+\infty$ for any $x \in(0, L)$. 


\section{Remarks.}

1. It is possible to give a physical meaning to the solvability (necessary and sufficient) assumption $f \in L^{1}\left(0, L: \delta_{\mathbf{a b}}\right)$. For instance, for the Dirichlet case it means that the momentum function of the shear stress at any interior point $x$ with respect the two extremes must be an integrable function.

2. Theorem 1 extends many previous works in the literature (see, e.g. [14], [2], [20] and other references presented in [8]). We also mention that the above versions in the literature on the weak maximum principle (valid under weaker conditions than the above (BC) have a non-quantitative version. Estimate (1) is new in the literature. It seems possible to extend the above result to the case of several dimensions but restricted to balls and under symmetry conditions on $f$. The maximum principle is false on some ellipsoidal domains (see [3] and the conjecture by Hadamard [15] in 1908 was firstly proved in [12]).

3. The existence result holds also in the more general class of Radon measures $f \in M\left(0, L: \delta_{\mathbf{a b}}\right)$ : something very useful to justify the engineers study in with the weight on the beam is concentrated at isolated points. Notice that although the usual Radon measure space (without weight) $M(0, L)$ is a subset of the dual space $H^{-2}(0, L)$ it is not always true that the duality $\langle f, \zeta\rangle_{H^{-2}(0, L), H_{0}^{2}(0, L)}$ coincides with the $\langle f, \zeta\rangle_{M(0, L), C^{0}([0, L])}=\int_{0}^{L} \zeta(x) d f$ duality.

\section{Perturbed operators in $L^{1}\left(\Omega, \delta_{\mathbf{a b}}\right)$.}

Many extensions of the above theorem are possible. For instance, the nonlinear problem

$$
(N L S P)\left\{\begin{array}{l}
\frac{d^{4} u}{d x^{4}}+\beta(u)=\gamma(u)+f(x) \\
+ \text { boundary conditions }(B C),
\end{array} \quad x \in \Omega=(0, L),\right.
$$

arises in many different frameworks: the linear case $\beta(u)=k u$ (and $\gamma \equiv 0$ ) corresponds to the so called elastic beam ([3], [15]). Monotone non decreasing functions $\beta(u)$ were used in [17] in the modeling of suspension bridges. A quite curious fact $([18],[16])$ : the strong maximum principle for the linear equation $\frac{d^{4} u}{d x^{4}}+k u=f(x)$ and boundary conditions $a_{0}=b_{0}=a_{2}=b_{2}=1$ is only true for $k \in\left(-k_{0}, k_{1}\right)$, for some $k_{0}, k_{1}>0$ depending on $L$. This also holds for the case of Dirichlet conditions: the associated Green function $G(x, y)$ can be explicitly built (for instance by means of the use of Mapple: see [8]) and it can be shown that if $k$ is large enough then $G\left(x_{0}, y_{0}\right) \geq 0$ for some $\left(x_{0}, y_{0}\right) \in[0, L]^{2}$.

Estimate (1) is quite "universal" as we can prove it for the solution of many related nonlinear problems. For instance, we have:

Theorem 2. For any $\beta$ maximal monotone graph of $R^{2}$ and any constant $\omega>\frac{1}{24 L^{4}}$ there exists a unique function $u$, with $\frac{d^{4} u}{d x^{4}} \in L^{1}\left(0, L: \delta_{\mathbf{a b}}\right)$ and $\beta\left(\frac{d^{4} u}{d x^{4}}\right) \in L^{1}\left(0, L: \delta_{\mathbf{a b}}\right)$, solution of the equation

$$
\beta\left(\frac{d^{4} u}{d x^{4}}\right)+\frac{d^{4} u}{d x^{4}}+\omega u=f(x)
$$


and satisfying $(D B C)$. Moreover, if $\widehat{u}$ is the solution for $\widehat{f}$, we have

$$
24 L^{4}\|u-\widehat{u}\|_{L^{1}\left(0, L: \delta_{\mathbf{a b}}\right)} \leq\|f-\widehat{f}\|_{L^{1}\left(0, L: \delta_{\mathbf{a b}}\right)} .
$$

Idea of the proof of Theorem 2. The operator $A: D(A) \rightarrow L^{1}\left(0, L: \delta_{\mathbf{a b}}\right)$ given by $A u=\frac{d^{4} u}{d x^{4}}$ if $u \in D(A)$ with $D(A)=\left\{u \in L^{1}\left(0, L: \delta_{\mathbf{a b}}\right) \cap C^{3}([0, L])\right.$ : $\frac{d^{4} u}{d x^{4}} \in L^{1}\left(0, L: \delta_{\mathbf{a b}}\right)$ and $u$ satisfies the $\left.(D B C)\right\}$ satisfies that $\exists C>0$ such that $C\|u\|_{L^{1}\left(0, L: \delta_{\mathbf{a b}}\right)} \leq\|A u\|_{L^{1}\left(0, L: \delta_{\mathbf{a b}}\right)}$ for all $u \in D(A)$. So, its inverse operator $J=A^{-1}$, satisfies that $J+C^{-1} I$ is accretive (and also $I-J$ when $C>1$ ): see [BeCrPaz]. Then, in particular, for any accretive operator $B$ on $L^{1}\left(\Omega, \delta_{\mathbf{a b}}\right)$ and for any $\lambda>0$ and $f \in L^{1}\left(0, L: \delta_{\mathbf{a b}}\right)$ the problem $\lambda\left(J w+B w+\frac{1}{C} w\right)+w=f$ has at most one solution $w \in L^{1}\left(\Omega, \delta_{\mathbf{a b}}\right)$ and we have the continuous dependence estimate $24 L^{4}\|u-\widehat{u}\|_{L^{1}\left(0, L: \delta_{\mathbf{a b}}\right)} \leq\|w-\widehat{w}\|_{L^{1}\left(0, L: \delta_{\mathbf{a b}}\right)} \leq\|f-\widehat{f}\|_{L^{1}\left(0, L: \delta_{\mathbf{a b}}\right)}$. The existence part is obtained firstly by truncating $f$ and by passing to the limit after using the a priori estimate mentioned before.

In general the operator $A$ is not T-accretive in $L^{1}\left(0, L: \delta_{\mathbf{a b}}\right)$, nevertheless, concerning the associated parabolic problem it is possible to show the following positivity result (which improves [13] for the one-dimensional case):

Theorem 3 (eventual positivity) Let $f \in L_{\text {loc }}^{1}\left(0,+\infty: L^{2}(0, L)\right)$ with $\frac{\partial f}{\partial t} \in$ $L^{1}\left(0,+\infty: L^{2}(0, L)\right)$ be such that $f(t, x) \rightarrow f_{\infty}(x)$ in $L^{2}(0, L)$ as $t \rightarrow+\infty$, with $f_{\infty}(x) \geq 0, f_{\infty} \neq 0$. Then, for any $u_{0} \in H^{4}(0, L) \cap H_{0}^{2}(0, L)$ and for any $\varepsilon>0$ small enough there exist a time $T_{\varepsilon} \geq 0$ such that the mild solution $u \in C\left([0,+\infty): L^{2}(0, L)\right)$ of the parabolic problem

$$
(H P)\left\{\begin{array}{cr}
\frac{\partial u}{\partial t}+\frac{\partial^{4} u}{\partial x^{4}}=f(t, x) & t \in(0, T), x \in(0,+\infty), \\
u(t, 0)=0, \quad u(t, L)=0, & t \in(0,+\infty), \\
\frac{\partial u}{\partial x}(t, 0)=0 \quad \frac{\partial u}{\partial x}(t, L)=0, & x \in(0, L),
\end{array}\right.
$$

satisfies that $u(t, x) \geq C\left(\left\|f_{\infty}\right\|_{L^{1}\left(0, L: \delta_{\mathbf{a b}}\right)}-\varepsilon\right) \delta_{\mathbf{a b}}(x)>0$ for any $t \geq T_{\varepsilon}$ and for any $x \in(0, L)$.

Idea of the proof. It is enough to use that $u(t, x) \rightarrow u_{\infty}(x)$ in $W^{2, \infty}(0, L)$ as $t \rightarrow+\infty$ (apply Theorem 3.9 of [Bre72]) with $u_{\infty}(x)$ given as the unique solution of $(S P)$ and $(D B C)$ with $f_{\infty}$ as right hand side and to apply the strong maximum principle b) of Theorem1.

Remark 7. The parabolic problem corresponds to the so called "quasistatic" problem associated to the Euler-Bernoulli transient hyperbolic problem (with a possible damping term) $\rho \frac{\partial^{2} u}{\partial t^{2}}+\mu \frac{\partial u}{\partial t}+\frac{\partial^{4} u}{\partial x^{4}}=f(t, x)$ which gives the dynamics decay when $\lambda$ is large enough.

Added in proof. After the obtention of the rsults of this Note, lectured by the author on September 16th, 2010, in the meeting "The Eleventh International Conference Zaragoza-Pau on Applied Mathematics and Statistics", Jaca (Spain) the author was informed by J.M. Rakotoson of the paper [1] in which the authors study the case of Dirichlet boundary conditions on a ball of $\mathbb{R}^{n}$ with $n \geq 2$ and 
under symmetry conditions on the data. Some of their results are close to conclusions b) and c) of Theorem 1 but the methos of proof are different and, as indicated before, the conclusions of Theorem 1 remains valid for the many other boundary conditions (for instance for the case of Dirichlet conditions at $x=0$ and Neumann at $x=L$ ) which cannot be related to formulations obtained trough radially symmetry solutions on balls of higher dimensions.

Acknowledgments. The author thanks Jean-Michel Rakotoson for indicating reference [1]. This research was partially supported by the project ref. MTM200806208 of the DGISPI (Spain) and the Research Group MOMAT (Ref. 910480) supported by UCM, and the ITN "FIRST" of the Seventh Framework Programme of the European Community's (grant agreement number 238702).

\section{References}

[1] I. Bachar, H. Māagli, S. Masmoudi, and M. Zribi, Estimates for the Green function and singular solutions for polyharmonic nonlinear equation, Abstract and Applied Analysis 2003 (2003), no. 12, 715741.

[BeCrPaz] Ph. Benilan, M. G. Crandall, and A. Pazy, Nonlinear Evolution Governed by. Accretive Operators. Book Manuscript, Besançon, France, 2001.

[2] F. Bernis, "On some nonlinear singular boundary value problems of higher order," Nonlinear. Analysis: Theory, Methods \& Applications, vol. 26, no. 6, pp. 1061-1078, 1996.

[3] T. Boggio, Sulle funzioni di Green d'ordine m, Rendiconti del Circolo Matematico di Palermo, Serie II 20 (1905), 97-135.

[Bre72] H. Brezis, Opérateurs maximaux monotones et semigroupes de contractions dans les espaces de Hilbert, North-Holland, Amsterdam 1972.

[4] H. Brezis, T. Cazenave, Y. Martel, A. Ramiandrisoa, Blow up for $u_{t}-\Delta u=g(u)$ revisited. Advance in Diff. Eq. 1, (1996), 73-90.

[5] H. Brezis, X. Cabré, Some simple nonlinear PDE's without solutions, Boll. Unione Mat. Ital. 1-B (1998), 223-262

[6] S. N. Chow, D. R. Dunninger and A.Lasota, A maximum principle for fourth order ordinary differential equations, J. Differential Equations, 14, 101-105, (1973).

[7] M.G. Crandall and L. Tartar, Some relations between nonexpansive and order preserving maps, Proc. AMS 78 (3) (1980) 385-390.

[8] J.I. Díaz, Very weak solutions of the beam and other higher order equations. To appear. 
[9] J.I. Díaz, J.M. Rakotoson, On the differentiability of very weak solutions with right-hand side data integrable with respect to the distance to the boundary, J. Functional Analysis, 257, (2009), 807-831.

[10] J.I.Díaz, J.M. Rakotoson, On very weak solutions of semi-linear elliptic equations in the framework of weighted spaces with respect to the distance to the boundary, Discrete and Continuous Dynamical Systems, 273 (2010) 1037-1058.

[11] J.I. Díaz, J. Hernandez, J.M. Rakotoson, Positive very weak solutions of singular semilinear problems. To appear

[12] R.J. Duffin, On a question of Hadamard concerning superbiharmonic functions, J. Math. Phys. 27 (1949), 253-258.

[13] F. Gazzola, H.C. Grunau, Some new properties of biharmonic heat kernels, Nonlinear Analysis 70, 2009, 2965-2973

[14] C.P. Gupta, Existence and uniqueness theorems for the bending of an elastic beam equation, Applicable Analysis 26 (1988), 289-305.

[15] J. Hadamard,,Memoire sur le probleme d'analyse relatif a l'equilibre des plaques elastiques incastrees, Memoires presentées par divers savants a l'Academie des Sciences, Vol. 33 (1908), 1-128.

[16] B. Kawohl and G. Sweers, On 'anti'-eigenvalues for elliptic systems and a question of McKenna and Walter, Indiana U. Math. J. 51, (2002), 1023-1040.

[17] P. J. McKenna and W. Walter, Nonlinear oscillations in a suspension bridge, Arch. Rational Mech. Anal., 98 (1987), 167-177.

[18] J. Schröder, Zusammenh"angende Mengen inverspositiver Differentialoperatoren vierter

Ordnung. (German) Math. Z. 96 (1967), 89-110.

[19] I. Stakgold, Green's Functions and Boundary Value Problems, Wiley, New York (1998)

[20] Q. Yao and Y. Li, Solution and positive solution to nonlinear cantilever beam equations, J. Southewest Jiaotong Univ. English Ed. 16 (2008), pp. 51-54. 